\title{
Lateral vibration effects in atomic-scale friction
}

\author{
R. Roth,,$^{1,2}$ O. Y. Fajardo, ${ }^{3}$ J. J. Mazo, ${ }^{3}$ E. Meyer, ${ }^{4}$ and E. Gnecco ${ }^{5}$ \\ ${ }^{1}$ Climate and Environment Physics, Physics Institute, University of Bern, Bern, Switzerland \\ ${ }^{2}$ Oeschger Centre for Climate Change Research, University of Bern, Bern, Switzerland \\ ${ }^{3}$ Departamento de Física de la Materia Condensada and Instituto de Ciencia de Materiales de Aragón, \\ CSIC-Universidad de Zaragoza, 50009 Zaragoza, Spain \\ ${ }^{4}$ Department of Physics, University of Basel, Klingelbergstrasse 82, 4056 Basel, Switzerland \\ ${ }^{5}$ Instituto Madrileño de Estudios Avanzados en Nanociencia, IMDEA Nanociencia, 28049 Madrid, Spain
}

(Received 24 September 2013; accepted 8 February 2014; published online 24 February 2014)

\begin{abstract}
The influence of lateral vibrations on the stick-slip motion of a nanotip elastically pulled on a flat crystal surface is studied by atomic force microscopy measurements on a $\mathrm{NaCl}(001)$ surface in ultra-high vacuum. The slippage of the nanotip across the crystal lattice is anticipated at increasing driving amplitude, similarly to what is observed in presence of normal vibrations. This lowers the average friction force, as explained by the Prandtl-Tomlinson model with lateral vibrations superimposed at finite temperature. Nevertheless, the peak values of the lateral force, and the total energy losses, are expected to increase with the excitation amplitude, which may limit the practical relevance of this effect. @ 2014 AIP Publishing LLC. [http://dx.doi.org/10.1063/1.4866427]
\end{abstract}

Developing strategies for lowering friction is a key issue for proper functioning of micro- and nano-electromechanical systems (MEMS and NEMS). In this context, traditional lubricants cannot be used, since the viscosity of mineral oils dramatically increases when the lubricant molecules are confined into nanometer-sized interstices. ${ }^{1}$ Different approaches, such as mechanical excitations, need to be explored. Ultrasonic vibrations have been used for years to modify the frictional behavior of materials at a macroscopic scale ${ }^{2}$ and their application at the nanoscale looks quite promising. ${ }^{3-7}$ Here, we are particularly interested in sharp asperities sliding on atomically flat surfaces. In this case, exciting mechanical resonances of the nano-junctions formed while sliding can be a valid method to reduce friction. This was shown by Socoliuc et al. ${ }^{5}$ in atomic-scale atomic force microscopy (AFM) experiments on alkali halide surfaces in ultra-high vacuum (UHV) and by Lantz et al., ${ }^{6}$ who succeeded in preventing the abrasive wear of a silicon tip sliding over several hundred meters following this strategy. The state of "dynamic superlubricity" so-achieved was also exploited to acquire lattice-resolved friction force maps of crystal surfaces without damaging the samples. ${ }^{8}$ Note that in the previous experimental works, the actuation was applied perpendicular to the sliding plane. Friction reduction was also predicted theoretically when mechanical oscillations are induced parallel to this plane. ${ }^{9}$ However, although this effect was observed in a series of macroscopic measurements based on a tribometer, ${ }^{10}$ the variation of the friction features on the atomic scale has not been documented so far.

In this work, we present AFM measurements in UHV complementing the results in Ref. 5, where the effect of normal excitations was investigated by applying an ac voltage between the tip and a counter electrode on the backside of an insulating $\operatorname{KBr}(001)$ surface. Here, lateral vibrations of the probing tip are induced by shaking a piezo-element attached to the cantilever sensor of the AFM at a frequency corresponding to the torsional resonance of the cantilever in contact with the sample surface. An alkali halide crystal, i.e., the $\mathrm{NaCl}(001)$ surface, was chosen as a model system for the measurements. Similar to the results presented in Ref. 5, the average friction on the probing tip decreases at increasing driving amplitude, which can be explained with the classical Prandtl-Tomlinson (PT) model for atomic-scale friction. ${ }^{11-17}$ However, this may not be the case for the peak values of the friction force, which are numerically expected to increase slightly when ultralow values of the average friction are approached.

The $\mathrm{NaCl}(001)$ sample surface was cleaved in a UHV chamber and analyzed using a home-built $\mathrm{AFM}^{18}$ and a silicon tip attached to an elastic cantilever with normal spring constant $k_{N}=0.08 \mathrm{~N} / \mathrm{m}$, and torsional spring constant $k_{T}=53 \mathrm{~N} / \mathrm{m}$ (Nanosensors PPP-CONT). The cantilever torsion is related to the (lateral) friction force experienced by the tip by standard formulas of continuum mechanics. ${ }^{19}$ The thermal noise power spectrum of the torsional vibrations of the cantilever in contact with the $\mathrm{NaCl}(001)$ surface is shown in Fig. 1. The resonance frequency of the system is found at $f_{t 0}=180.3 \mathrm{kHz}$. Note that another resonance peak $f_{n 1}=171.5 \mathrm{kHz}$ is observed, corresponding to the second flexural mode of the cantilever in contact. The relative sharpness of the resonance curves (widths at half-maximum of about $2 \mathrm{kHz}$ ) allows us to exclude any overlap between the two resonance modes. To get the optimum friction reduction, a phase-locked loop (PLL) was used to track the torsional resonance peak and excite the cantilever at the frequency $f_{t 0}=180.3 \pm 0.5 \mathrm{kHz}$ (gray bar in Fig. 1) while scanning the sample surface. This induced lateral vibrations of the probing tip.

Fig. 2 shows two friction loops acquired while shaking the cantilever at the frequency $f_{t 0}$ and without doing that. Note that the lateral force is significantly smoothed out due to sampling. In absence of vibrations, a typical sawtooth pattern is observed. The tip is pinned to a certain site on the surface lattice till the angle of torsion of the cantilever reaches a critical value, and the tip suddenly slips into a nearby location. When the lateral vibrations are excited, the sawtooth pattern is shrunk, i.e., the trace and retrace curves become closer till they completely overlap if the excitation amplitude 


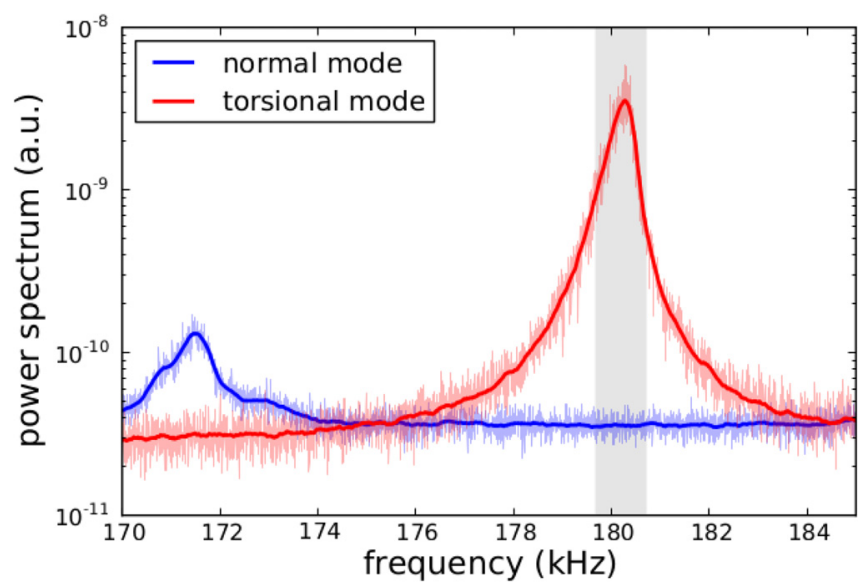

FIG. 1. Thermal noise power spectrum of the normal (blue curve) and torsional vibrations (red curve) of the AFM sensor used in the experiment in contact with a $\mathrm{NaCl}(001)$ surface in UHV.

is high enough (dark blue curves in Fig. 2). In this case, the friction force $F_{\text {fric }}$, that is, the lateral force averaged over one or multiple oscillation cycles, becomes negligible. Note that the smoothing applied to $F_{\text {fric }}$ is much shorter than the washboard frequency. The inset in Fig. 2 shows also that the complete slip of the tip towards a new equilibrium position is accompanied by a series of back-and-forth jumps induced by the thermal vibrations occurring at the finite temperature ( $T=300 \mathrm{~K})$ of the experiment. The excitation amplitude dependence of the friction force, averaged over the stick-slip movement across the crystal lattice (i.e., of the area enclosed by the friction loops, divided by the distance travelled by the clamped end of the cantilever support), is shown in Fig. 3. This force is denoted $\left\langle F_{\text {fric }}\right\rangle$, to distinguish it from $F_{\text {fric }}$, which is only averaged over a few oscillation cycles. The force $\left\langle F_{\text {fric }}\right\rangle$ decreases gradually, as observed when normal oscillations are applied. ${ }^{5}$

To interpret our results, we have run analytical and numeric calculations based on the PT model. Here, a point mass representing the apex of the probing tip is pulled across a periodic potential of amplitude $U_{0}$, simulating the interaction with the surface lattice, using a spring of stiffness $k$. The

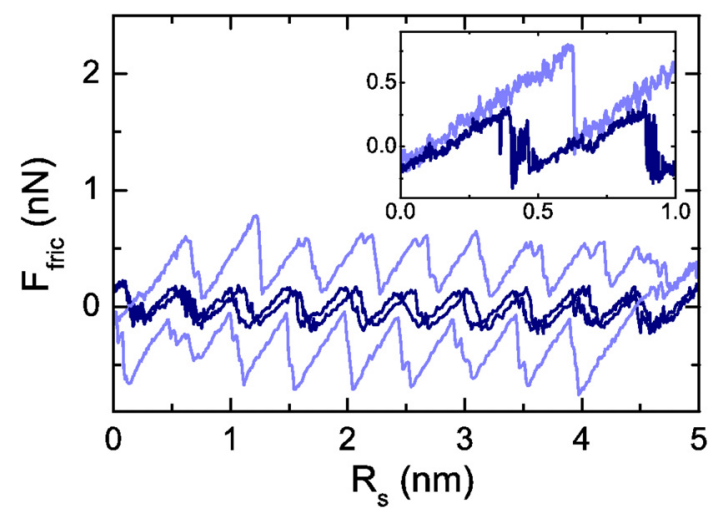

FIG. 2. Atomic-scale friction force loops measured on $\mathrm{NaCl}(001)$ in $\mathrm{UHV}$ at room temperature with (dark blue) and without (light blue) lateral vibrations. $R_{s}=v_{s t}$ is the cantilever position averaged over the vibrations. The driving amplitude of the $a c$ voltage is $U_{\text {exc }}=100 \mathrm{mV}$ and the frequency $f=180.3$ $\mathrm{kHz}$ corresponds to the torsional resonance in Fig. 1. Each data point is averaged over 180 oscillations cycles (over 36 cycles in the inset, where only the forward signal is shown). The normal force and velocity values are $F_{N}=4.9 \mathrm{nN}$ and $v_{s}=10 \mathrm{~nm} / \mathrm{s}$ in both cases.

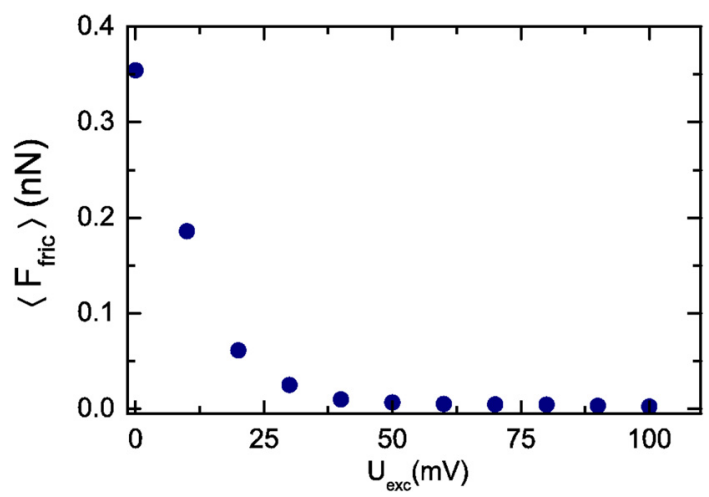

FIG. 3. Measured average friction force as a function of the amplitude of the $a c$ voltage inducing the lateral oscillations.

clamped end of the spring moves with the time $t$ as $R(t)=v_{s} t+A \sin (2 \pi f t)$, where $A$ and $f$ are the amplitude and frequency of the lateral vibrations, and $v_{s}$ is the scan velocity. A random noise force $\xi(t)$, which is related to the temperature $T$ by the fluctuation-dissipation relation, is also added to the forces acting on the tip, in order to reproduce thermal effects. A lateral force loop resulting from model values consistent with the experimental parameters is shown in Fig. 4. Note that the value of the effective mass was fixed to $7.7 \times 10^{-16} \mathrm{~kg}$, corresponding to a resonance frequency of $5.6 \mathrm{MHz}$ and the simulations were performed at the critical damping. The average friction signal is indeed reduced when the lateral vibrations are included and the trace and retrace signals tend to overlap, as in the AFM experiments. Back-and-forth jumps are also present, as seen in the inset of Fig. 4.

The variation of the average friction force with the driving amplitude is plotted in Fig. 5, at both zero and room temperature. We observe that the simulated points obtained at $T=0 \mathrm{~K}$ are well-fitted by the expression

$$
\begin{aligned}
\left\langle F_{\text {fric }}\right\rangle(A) \simeq & \frac{k a}{2 \pi}\left\{\eta-\pi\left(1+\frac{2 A}{a}\right)\right. \\
& \left.+\frac{4}{3} \sqrt{\frac{\pi}{\eta}}\left[\left(1+\frac{A}{a}\right)^{3 / 2}-\left(\frac{A}{a}\right)^{3 / 2}\right]\right\},
\end{aligned}
$$

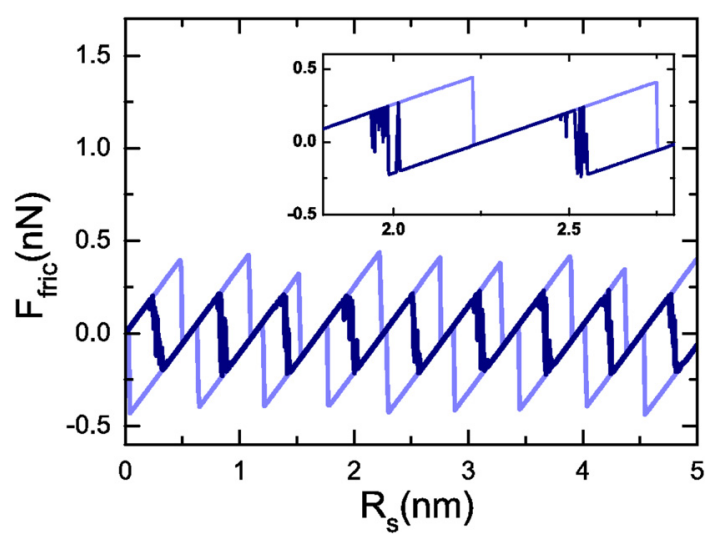

FIG. 4. Simulated friction force loops with (dark blue) and without (light blue) superimposed lateral oscillations. Parameter values: $a=0.564 \mathrm{~nm}$, $U_{0}=0.38 \mathrm{eV}, k=0.95 \mathrm{~N} / \mathrm{m}, A=0.6 a, f=180.3 \mathrm{kHz}, \quad v_{s}=10 \mathrm{~nm} / \mathrm{s}$, and $T=300 \mathrm{~K}$. The inset shows the back-and-forth jumps accompanying the transition from a lattice site to the next one. As in Fig. 2, the lateral force is averaged over 180 (36 in the inset) oscillation cycles. 


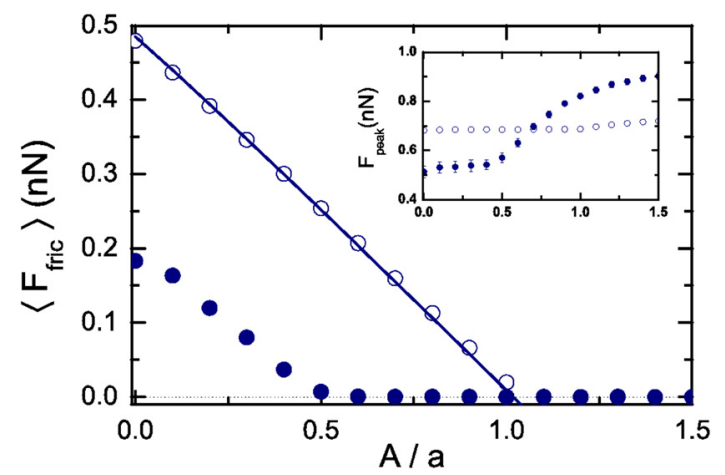

FIG. 5. Computed lateral force $\left\langle F_{\text {fric }}\right\rangle$, as averaged across the crystal lattice, at increasing lateral driving amplitude. The results at $T=0 \mathrm{~K}$ (empty circles) are well-reproduced by the continuous line defined by the Eq. (1). The filled circles are obtained when $T=300 \mathrm{~K}$. The corresponding peak values of the instantaneous lateral force (not averaged) are shown in the inset. We show the mean peak force computed for 100 stick-slip cycles and the obtained error bars.

where $\eta=4 \pi^{2} U_{0} /\left(k a^{2}\right)$. Equation (1) is obtained by power series expansion, as detailed in Ref. 20. As shown in Fig. 5, it predicts an almost linear decrease of friction with the oscillation amplitude in the range of parameters that we considered. This conclusion is partially modified by the presence of thermal vibrations. Including the thermal noise term $\xi(t)$ in the equations of motion, the $\left\langle F_{\text {fric }}\right\rangle$ vs. $A$ curve is lowered and slightly bent, as shown by the filled circles in Fig. 5 . This is not surprising, since thermal vibrations are also quite effective in reducing friction on the nanoscale. ${ }^{13,16} \mathrm{~A}$ comparison of Figs. 3 and 5 shows that an excitation of $50 \mathrm{mV}$
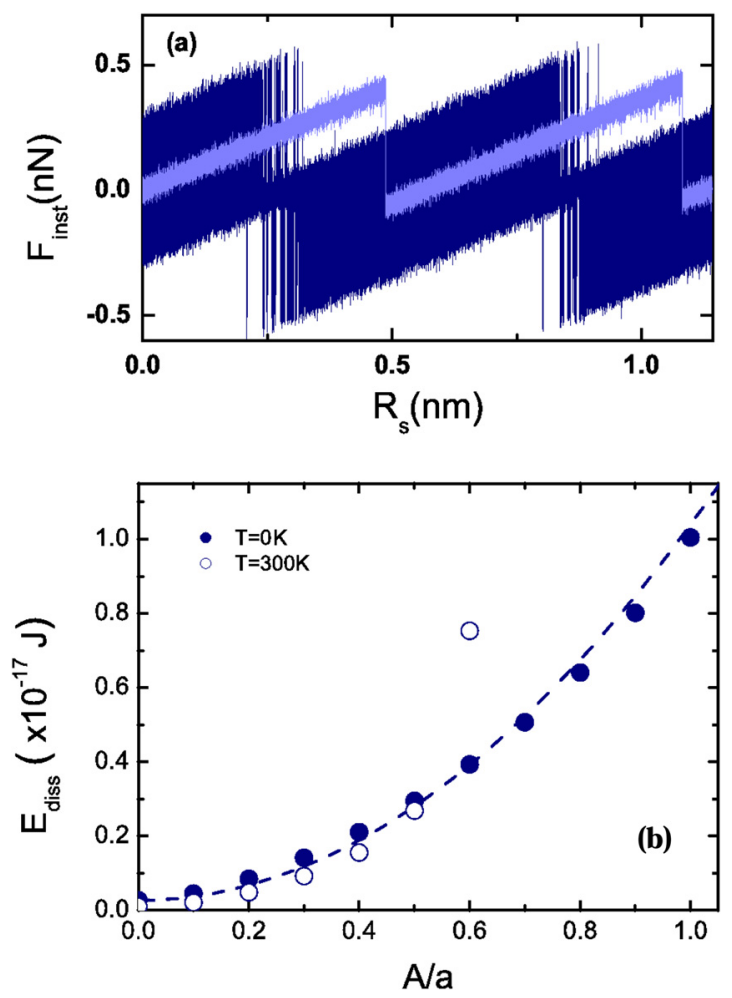

FIG. 6. (a) Simulated instantaneous variation of the lateral force with (dark blue) and without (light blue) superimposed lateral oscillations (the parameter values are the same as in Fig. 4). (b) Energy dissipation expected at different values of the excitation amplitude $A$ at $T=0 \mathrm{~K}$ (closed circles) and $T=300 \mathrm{~K}$ (open circles). The dashed curve shows a quadratic fit expected below the transition to the superlubric regime. applied to the piezo actuator leads to lateral oscillations of approximately half lattice constant of the cantilever support, suggesting a simple method for a rough calibration of the driving amplitude.

Our analysis would not be complete without considering the instantaneous variations of the lateral force $F_{\text {inst }}(t)=k[R(t)-x(t)]$, where $R(t)$ and $x(t)$ are the support and tip position, respectively. As seen in Fig. 6, this force is rapidly oscillating and we notice that its peak values do not vary significantly with the excitation amplitude although the average lateral force value $\left\langle F_{\text {fric }}\right\rangle$ decreases. In the simulations at room temperature, we even observe an increase of the peak force when the average force $\left\langle F_{\text {fric }}\right\rangle$ becomes negligible around $A \simeq 0.5 a$ (see inset of Fig. 5). Even more pronounced is the variation of the energy dissipation $E_{\mathrm{diss}}$, which is calculated as the power $F_{\text {inst }} \cdot d R / d t$, integrated over a lattice period. The energy loss $E_{\text {diss }}$ is found to increase with the amplitude $A$, as shown in Fig. 6(b). When the ultralow average friction regime is entered (when $A \simeq a$ at $T=0 \mathrm{~K}$ or $A \simeq 0.5 a$ at $T=300 \mathrm{~K}$ ), the increase of $E_{\text {diss }}$ becomes steeper.

In summary, we have performed atomic-scale friction measurements on a $\mathrm{NaCl}(001)$ surface in which lateral vibrations of the nanoprobe are excited while sliding on the sample surface. As a result, a continuous decrease of the average friction force with the driving amplitude is observed. The experimental results are supported by numeric calculations based on the Prandtl-Tomlinson model including thermal vibrations. As a drawback, our simulations also predict that, oppositely to the average friction force, the energy dissipation in the system may become significant when the probe is vibrated, which may seriously limit the application of our results to reduce friction and wear in nanodevices. A thorough analysis of the lateral force signal using high bandwidth, as done by Maier et al. in absence of external excitations, ${ }^{21}$ may help to shed light on this important issue. Applications to specific configurations such as cantilevers oscillating in the pendulum geometry in close proximity to a solid surface ${ }^{22,23}$ are also possible.

We thank Dr. Thilo Glatzel and Dr. Shigeki Kawai for technical assistance. O.Y.F. and J.J.M. acknowledge the financial support from Spanish MINECO through Project No. FIS2011-25167, cofinanced by FEDER funds. O.Y.F. acknowledges the financial support from FPU grant by Ministerio de Ciencia e Innovación of Spain. E.G. acknowledges the financial support from Spanish MINECO through Project No. MAT2012-34487. E.M. acknowledge the Swiss National Science Foundation, the Swiss Nanoscience Institute and the Commission for Technology and Innovation. Authors also acknowledge the COST Action MP1303.

${ }^{1}$ Y. Z. Hu and S. Granick, Tribol. Lett. 5, 81 (1998).

${ }^{2}$ See, e.g., A. Akay, J. Acoust. Soc. Am. 111, 1525 (2002) and references therein.

${ }^{3}$ F. Dinelli, S. K. Biswas, G. A. D. Briggs, and O. V. Kolosov, Appl. Phys. Lett. 71, 1177 (1997).

${ }^{4}$ G. Behme and T. Hesjedal, J. Appl. Phys. 89, 4850 (2001).

${ }^{5}$ A. Socoliuc, E. Gnecco, S. Maier, O. Pfeiffer, A. Baratoff, R. Bennewitz, and E. Meyer, Science 313, 207 (2006).

${ }^{6}$ M. A. Lantz, D. Wiesmann, and B. Gotsmann, Nat. Nanotechnol. 4, 586 (2009). 
${ }^{7}$ W. M. van Spengen, G. H. C. J. Wijts, V. Turq, and J. W. M. Frenken, J. Adhes. Sci. Technol. 24, 2669 (2010).

${ }^{8}$ E. Gnecco, A. Socoliuc, S. Maier, J. Gessler, T. Glatzel, A. Baratoff, and E. Meyer, Nanotechnology 20, 025501 (2009).

${ }^{9}$ Z. Tshiprut, A. Filippov, and M. Urbakh, Tribol. Int. 40, 967 (2007).

${ }^{10}$ V. L. Popov, J. Starcevic, and A. E. Filippov, Tribol. Lett. 39, 25 (2010).

${ }^{11}$ L. Prandtl, Z. Angew. Math. Phys. 8, 85 (1928).

${ }^{12}$ E. Gnecco, R. Bennewitz, T. Gyalog, C. Loppacher, M. Bammerlin, E. Meyer, and H. J. Güntherodt, Phys. Rev. Lett. 84, 1172 (2000).

${ }^{13}$ Y. Sang, M. Dube, and M. Grant, Phys. Rev. Lett. 87, 174301 (2001).

${ }^{14}$ M. Reguzzoni, M. Ferrario, S. Zapperi, and M. C. Righi, Proc. Natl. Acad. Sci. U.S.A. 107, 1311 (2009).

${ }^{15}$ O. Y. Fajardo and J. J. Mazo, Phys. Rev. B 82, 035435 (2010).

${ }^{16}$ L. Jansen, H. Hölscher, H. Fuchs, and A. Schirmeisen, Phys. Rev. Lett. 104, 256101 (2010).
${ }^{17}$ M. H. Müser, Phys. Rev. B 84, 125419 (2011).

${ }^{18}$ L. Howald, E. Meyer, R. Lüthi, H. Haefke, R. Overney, H. Rudin, and H. J. Güntherodt, Appl. Phys. Lett. 63, 117 (1993).

${ }^{19}$ Springer Handbook of Nanotechnology, edited by B. Bhushan (Springer, 2007).

${ }^{20}$ O. Y. Fajardo, E. Gnecco, and J. J. Mazo "Out-of-plane and in-plane actuation effects on atomic-scale friction," Phys. Rev. B (to be published); e-print arXiv: 1402.3576

${ }^{21}$ S. Maier, Y. Sang, T. Filleter, M. Grant, R. Bennewitz, E. Gnecco, and E. Meyer, Phys. Rev. B 72, 245418 (2005).

${ }^{22}$ M. Kisiel, E. Gnecco, U. Gysin, L. Marot, S. Rast, and E. Meyer, Nature Mater. 10, 119 (2011).

${ }^{23}$ M. Langer, M. Kisiel, R. Pawlak, F. Pellegrini, G. E. Santoro, R. Buzio, A. Gerbi, G. Balakrishnan, A. Baratoff, E. Tosatti, and E. Meyer, Nature Mater. 13, 173 (2014). 